\title{
Respuesta climática de Pinus oocarpa Schiede Ex Schetol en el Bosque La Primavera, Jalisco
}

\section{Climatic response of Pinus oocarpa Schiede Ex Schetol in the "La Primavera" Forest, Jalisco}

\author{
José Villanueva-Díaz*, Ernesto Alonso Rubio-Camachol, Álvaro Agustín Chávez-Durán', José Luis Zavala-Aguirre² \\ Julián Cerano-Paredes' y Aldo Rafael Martínez-Sifuentes' \\ Instituto Nacional de Investigaciones Forestales \\ Agrícolas y Pecuarias. CENID RASPA. Gómez \\ 2 Universidad Autónoma de Guadalajara. Escuela de
Biología. Zapopan, Jalisco, México.
} Palacio, Durango, México

\section{RESUMEN}

El Bosque La Primavera (BLP) es una reserva de importancia ecoturística y un proveedor de servicios ambientales para la metrópoli de Guadalajara. Sin embargo, las actividades humanas han alterado la estabilidad de sus ecosistemas. El objetivo del estudio fue analizar la variabilidad hidroclimática de la especie dominante Pinus oocarpa de alta sensibilidad climática. Cronologías de madera temprana (EWI), tardía (LWI) y de anillo total (RWI), con 165 años de extensión (1850-2014), se generaron para la especie en BLP. La serie residual EWI se correlacionó significativamente con la precipitación acumulada octubre-mayo $(\mathrm{r}=0.775, \mathrm{p}<0.000)$, de estaciones climáticas aledañas a la reserva y se utilizó para una reconstrucción de precipitación; asimismo, se encontró una asociación negativa, pero significativa, entre temperatura máxima promedio en enero-junio y la serie EWI $(\mathrm{r}=-0.622$, $\mathrm{p}<0.000)$.

La sequía más prolongada en la reconstrucción se detectó de 1909 a 1925, otras más como las de las décadas 1850, 1890, 1950,1990 y 2000 se encuentran presentes en otras reconstrucciones climáticas para Jalisco, lo que es indicativo de la influencia de fenómenos de circulación general como El Niño Oscilación del Sur (ENSO). El incremento en la temperatura máxima en las últimas tres décadas en el BLP ha afectado el crecimiento de la especie con una tendencia a reducirlo, situación que pone en riesgo la estabilidad de esta y de otras especies asociadas y, por ende, la provisión de servicios ecosistémicos a la metrópoli.

Palabras CLAVE: calentamiento global, dendrocronología, ENSO, Pinus oocarpa, sequía.

\begin{abstract}
The Bosque la Primavera (BLP) is a natural preserve that provides environmental services for the metropolitan area of Guadalajara. Human activities, however, have altered its ecological stability. The objective was to analyze the interannual climate variability of dominant conifer Pinus oocarpa, a climatesensitive species. Earlywood (EWI), latewood (LWI), and ring-width (RWI) chronologies 165 years in length (1850-2014) were developed for the BLP. The residual EWI chronology had a significant association $(r=0.775, \mathrm{p}<0.000)$ with seasonal October-May rainfall from neighbor weather stations, and a negative but significant response with January-June mean maximum temperature $(\mathrm{r}=-0.622, \mathrm{p}<0.000)$. The association between EWI and precipitation was used to build a linear model to reconstruct seasonal rainfall.

The most severe and prolonged drought in the reconstruction took place from 1909 to 1925, but additional dry periods were present, i.e., 1850's, 1890's, 1950 's, 1990's, and 2000's. Most of these droughts have been observed in previous reconstructions for the region, suggesting the influence of atmospheric circulatory patterns such as ENSO. The rise in maximum temperature for the last three decades in the Guadalajara metropolitan area has induced a radial growth decrease of $P$. oocarpa, probably triggered by an increase in greenhouse gases. Increased maximum temperatures and reduced growth of the species is threatening its ecological stability and may put in risk the provision of vital environmental services for this metropolitan area.
\end{abstract}

KEYWORDS: global warming, dendrochronology, ENSO, Pinus oocarpa, drought. 


\section{INTRODUCCIÓN}

Las áreas forestadas que rodean a las grandes urbes proveen de una serie de servicios ambientales, sin los cuales difícilmente estas existirían; sin embargo, la presión de los asentamientos humanos, conforme la población se incrementa, incide directamente sobre la estabilidad ecológica de los ecosistemas, a través de incendios intencionales, deforestación, presencia de lluvia ácida y pérdida de la biodiversidad, afectando de manera significativa la oferta de estos servicios (Dodds, Perkin y Gerken, 2013). Esta situación se presenta en el Bosque La Primavera (BLP), un área colindante con la ciudad de Guadalajara, Jalisco, y que en las últimas décadas ha sufrido una degradación progresiva debido, entre otros factores, a incendios intencionales y a cambios severos en el uso del suelo para incrementar la mancha urbana (Comisión Nacional de Áreas Naturales Protegidas [Conanp], 2000).

El BLP ha sido objeto de una serie de estudios relacionados con su biodiversidad, procesos sucesionales derivados de incendios, superficie afectada e impacto ecológico (Gallegos, González, Cabrera, Marcelli y Hernández, 2014; Huerta y Ibarra, 2014); sin embargo, no existen estudios específicos para analizar la influencia del clima en el incremento radial de especies que producen anillos de crecimiento anual. Esta información es relevante para determinar el impacto de disturbios de origen antropogénico y la influencia del clima; en particular, considerando el calentamiento global y la respuesta de las especies a este fenómeno, que, se indica, minimiza el nicho ecológico de coníferas y latifoliadas y amenazan la permanencia de algunas de ellas en el ecosistema (Baron et al., 2009).

El entendimiento del comportamiento histórico del clima analizado, a través de los anillos anuales de crecimiento de algunas especies arbóreas, permite determinar la variabilidad hidroclimática de alta y baja frecuencia más allá de los registros instrumentales disponibles, y los fenómenos climáticos de circulación general que la afectan y el potencial efecto de acciones antropogénicas en el comportamiento del clima actual. Los registros climáticos existentes difícilmente se extienden por más de 70 años $y$ tienen el inconveniente de su calidad y representatividad, particularmente en sitios elevados o de difícil acceso, donde no existen o se dispone de pocas estaciones climáticas en funcionamiento, pero que es indispensable su existencia, ya que son sitios donde se generan los servicios hidrológicos, de los cuales dependen grandes zonas metropolitanas como es el caso de Guadalajara, Jalisco, con una población de 4.8 millones en 2015 (Instituto Nacional de Estadística y Geografía [Inegi], 2015), pero que para el 2018 se estima un incremento a cinco millones de habitantes.

En este tenor, el desarrollo de series dendrocronológicas en el BLP contribuirá a generar información interanual y multianual del comportamiento del clima más allá de los registros instrumentales; y a determinar el impacto de eventos hidroclimáticos extremos, así como su frecuencia histórica y tendencias. Esta información es de suma importancia para analizar la relación entre el crecimiento de la vegetación y la variabilidad de estos factores climáticos, la dinámica del ecosistema y la producción de servicios ambientales con fines de planear su uso sustentable y la conservación de los mismos.

\section{OBJETIVOS}

El objetivo del presente estudio fue desarrollar una reconstrucción dendroclimática con Pinus oocarpa, especie dominante en el BLP. La reconstrucción permitirá analizar la variabilidad interanual y multianual del clima, más allá de los datos instrumentales, y determinar los fenómenos circulatorios que la afectan.

\section{MATERIALES Y MÉTODOS}

\section{Área de estudio}

El sito de estudio se ubica en el BLP, que es una zona forestal protegida localizada al oeste de la ciudad de Guadalajara, Jalisco, en las coordenadas geográficas extremas $20^{\circ} 37^{\prime} 59.25^{\prime \prime} \mathrm{N}-103^{\circ}$ 33' 35.37"O; 20॰ 37' 52.2” N-103' 33' 35.57”' O. Actualmente, es un área de protección de flora y fauna, enclavada en la región hidrológica Lerma-Chapala-Santiago y Ameca, la cual provee con más de 240 millones de metros cúbicos $\left(\mathrm{Mm}^{3}\right)$ de recursos hídricos y de otros servicios ambientales a la zona metropolitana de Guadalajara, Jalisco (Conanp, 2000).

La reserva posee dos tipos de clima: templado subhúmedo, $\mathrm{S}\left(\mathrm{w}_{1}\right)(\mathrm{w})$, y semicálido, (A)C $\left(\mathrm{w}_{1}\right)$ (w) (García, 2004); dominan las lluvias de verano y solo 5\% ocurren en invierno; la precipitación anual presenta un rango de $800 \mathrm{~mm}$ a $1000 \mathrm{~mm}$, con una temperatura media anual de $20.5^{\circ} \mathrm{C}$. Los suelos se clasifican morfológica- 
mente como regosoles y litosoles: los primeros derivados de tobas, pómez y riolita, ocurren en 92\% del área; mientras que los litosoles constituyen 8\% (Huerta y Ibarra, 2014). En general, los suelos son someros con una profundidad menor a $60 \mathrm{~cm}$, bajo contenido de materia orgánica $(<2 \%)$ y pH ácido $(<5.5)$.

Florísticamente, BLP forma parte de la Sierra Madre Occidental y Eje Neovolcánico Transversal y pertenece a la región fisiográfica de la Cordillera Neovolcánica. Posee diversos tipos de vegetación que se distribuyen a una elevación de $1400 \mathrm{~m}$ a $2225 \mathrm{~m}$, entre los que se encuentra el bosque de pino, bosque de pino-encino, bosque de encino y bosque tropical caducifolio, donde habitan más de1000 especies de plantas, 135 especies de aves, 29 especies de mamíferos, entre otras especies de reptiles (Conanp, 2000).

Uno de los problemas fundamentales que deteriora la estabilidad ecológica del BPL son los incendios frecuentes, muchos de ellos intencionales, que afectan los diversos tipos de vegetación, en particular de coníferas, donde según la intensidad ocasionan hasta $55 \%$ de mortandad, como fue el caso de los incendios registrados en 1984, 1987, 1995, 1998, 2005 у 2012 - estos últimos tres afectaron una superficie superior a 32000 ha, es decir, 88\% del BLP (Gallegos et al., 2014).

Cambios de uso del suelo, entre los que se encuentra el pastoreo intensivo, expansión de áreas agrícolas, talas clandestinas, aprovechamiento de bancos de material, apertura de áreas de recreación, construcción de caminos, entre algunos otros usos, han afectado la estabilidad ecológica de los tipos de vegetación presentes en la reserva, acelerando el proceso erosivo, alterando su dinámica y la ecología del paisaje, que es otro de los servicios ambientales intrínsecos de esta reserva, amén de ser el pulmón principal que oxigena el área metropolitana de Guadalajara.

\section{MÉTODOS}

El sitio de muestreo posee una fisiografía de lomerío con una vegetación de bosque de pino-encino y alta incidencia de incendios, donde el dosel dominante está formado por Pinus oocarpa, Quercus magnoliifolia, Quercus viminea, Clethra rosei y Acacia pennatula. Se ubica en las coordenadas geográficas $20^{\circ} 38^{\prime} 40.9^{\prime \prime}$ de latitud N, longitud de 103³5’9.8” OG y elevación de $1884 \mathrm{~m}$ snm (Fig. 1).

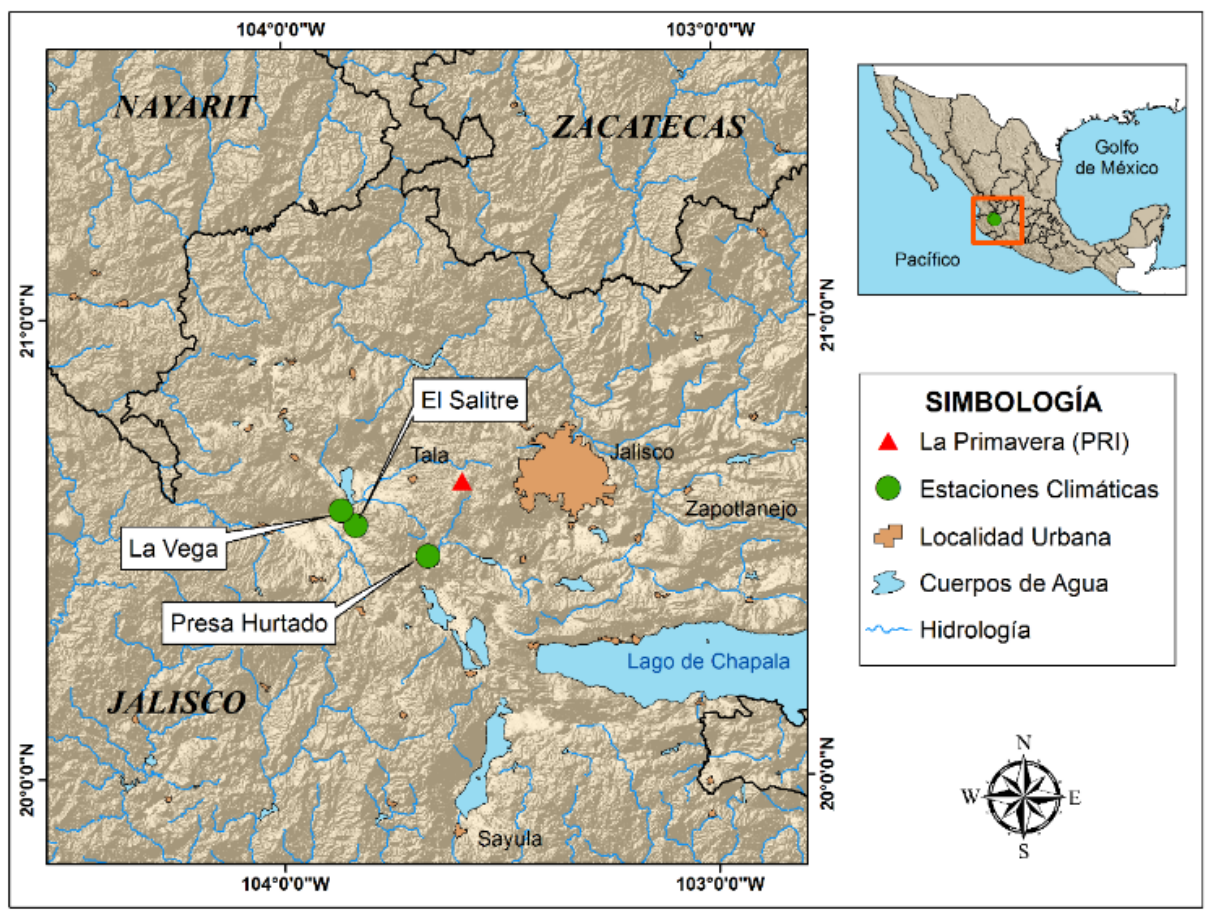

FIGURA 1. Ubicación geográfica del sitio de colecta de núcleos de crecimiento de $P$. oocarpa en el Bosque la Primavera, Jalisco. 
Árboles de Pinus oocarpa se ubicaron en sitios con pobre calidad de estación (suelos someros, baja fertilidad, escarpados) y con el menor disturbio posible. Se seleccionó un total de 41 árboles, a los cuales se les extrajo tres núcleos de crecimiento (virutas, incrementos) distribuidos en caras opuestas del fuste y perpendicular a la pendiente para evitar irregularidades en el crecimiento (problemas de liberación y compresión). Las muestras se procesaron con base a técnicas dendrocronológicas convencionales (Stokes y Smiley, 1968).

Para maximizar la información climática, el anillo total se dividió en dos bandas de crecimiento, madera temprana y madera tardía, cada una de ellas comúnmente influenciada en su desarrollo por condiciones climáticas distintas (Panshin y De Zeeuw, 1980). Las bandas individuales se midieron con un sistema de medición Velmex y las bases de medición se corrieron en COFECHA, que es un programa de control de calidad y útil para determinar problemas potenciales de fechado (Holmes, 1983). La base de datos de medición después de realizada la corrección de fechado ocasionada por problemas de anillos falsos, perdidos o por errores de asignación de fechas, se corrió en el programa ARSTAN, que estandariza las series de crecimiento y elimina información no deseable (ruido) debido a cuestiones biológicas y geométricas (Cook, 1987). Para este proceso se utilizó una doble estandarización, que se fundamente en las tendencias en crecimiento de la especie, donde la primera consistió en fijar a las series de crecimiento, curvas exponenciales negativas o rectas de regresión positivas o negativas; para la segunda estandarización, se ajustó una curva flexible (spline) con una rigidez de $67 \%$ y que preserva $50 \%$ de la varianza en las series (Cook, 1987), lo cual permite conservar en estas variaciones de baja frecuencia de origen climático. Los índices se obtuvieron por división.

Se generaron tres cronologías, madera temprana (EWI, por sus siglas en inglés), madera Tardía (LWI, por sus siglas en ingles) y de anillo total (RWI, por sus siglas en ingles), cada una con tres versiones de la cronología, que son la "Estándar", "Residual" y "Arstan", las cuales para su generación involucran diversos procesos estadísticos descritos a detalle en Cook (1987).

Estaciones climáticas e hidrométricas cercanas al sitio de colecta se obtuvieron tanto del Extractor Rápido de Información Climática (ERIC, 2013) como de la Base de Datos Hidrométrica de la Comisión Nacional del Agua (Banco Nacional de Datos de Aguas Superficiales [Bandas], 2006) (Tabla 1). Datos de precipitación acumulada mensual, temperatura mínima, máxima y media y volúmenes hidrométricos de las estaciones seleccionadas de manera individual o combinadas, se compararon con los índices EW, LW y RW en sus versiones estándar y residual mediante funciones de respuesta, para definir posibles relaciones significativas con crecimiento y desarrollar reconstrucciones históricas de las variables relacionadas significativamente (Fritts, 1991).

TABLA. 1. Serie dendrocronológica de la reserva La Primavera y estaciones climáticas e hidrométricas aledañas al sitio de colecta y consideras para definir la respuesta climática de P. oocarpa.

\begin{tabular}{|c|c|c|c|c|c|}
\hline Sitio & Lat.(grad) & Lon. (grad) & Elev. $(m)$ & Período & Tipo de cronologíal \\
\hline La Primavera (PRI) & 20.644 & 103.586 & 1884 & -------- & RW, EW, LW \\
\hline \multicolumn{6}{|c|}{ Estaciones Climáticas } \\
\hline La Vega & 20.583 & -103.867 & 1250 & 1954-2003 & \\
\hline Presa Hurtado & 20.483 & -103.667 & 1250 & 1947-2003 & \\
\hline \multicolumn{6}{|c|}{ Estaciones Hidrométricas } \\
\hline Santa Rosa II & 20.914 & -103.718 & 748 & 1958-2011 & \\
\hline San Cristobal II & 21.038 & -103.429 & 870 & 1960-2011 & \\
\hline El Salitre & 20.533 & -103.867 & 1247 & 1962-2011 & \\
\hline
\end{tabular}

${ }^{3} \mathrm{PR}$ : Clave de la serie dendrocronológica para el BLP; RW= Anillo Total, EW= Madera Temprana, LW= Madera Tardía 
Para determinar la influencia de fenómenos de circulación atmosférica en el comportamiento del clima y en el crecimiento radial del arbolado, los índices dendrocronológicos se compararon con índices de fenómenos atmosféricos circulatorios como El Niño Oscilación del Sur (ENSO, por sus siglas en ingles), Oscilación Decadal del Pacífico (PDO, por sus siglas en inglés), Oscilación Multidecadal del Atlántico (AMO, por sus siglas en inglés) e índices de sequía reconstruidos e instrumentales del índice de Sequía de Severidad de Palmer (PDSI, por sus siglas en inglés); así como con niveles anuales recuperados del Lago de Chapala, cuyos volúmenes aportados al mismo mediante una serie de afluentes, amalgaman los efectos de la variabilidad hidroclimática que caracteriza a las cuencas productoras de agua en la zona, de la cual forma parte el BLP.

Los periodos de sequía o períodos húmedos detectados en la cronología se compararon con otras series dendrocronológicas y reconstrucciones climáticas desarrolladas para la región y para otras zonas del país, con el fin de analizar el efecto de la variabilidad climática y los impactos originados en la producción de alimentos y en la estabilidad social y económica de la población.

\section{Resultados}

\section{Incremento radial anual}

El incremento radial anual de los árboles seleccionados se ajustó a una exponencial negativa, crecimiento típico de coníferas en el norte y centro de México, atribuido a efectos biológicos y aun radio cada vez mayor (Fritts, 1976). Las series de crecimiento anual $(\mathrm{mm})$, muestran alta variabilidad interanual, con un crecimiento superior en la parte interna (centro del árbol) e inferior en el exterior (pegado a la corteza) (Fig. 2).

\section{Serie dendrocronológica}

La cronología de anillo total de P. oocarpa se extiende de 1850 a 2014 (165 años) (Fig. 3); la versión estándar de la cronología mostró una inter-correlación entre series (medida de la intensidad de la señal común entre series) de 0.506, que supera 0.3281(p < 0.01), considerado el valor de referencia (Holmes, 1983); sensibilidad media (cambio relativo en el ancho de anillos de un año al siguiente) de 0.322 , valor considerado aceptable con fines de reconstrucción; autocorrelación de primer orden (grado en el que el grosor del anillo actual es influenciado por el crecimiento del año previo) de 0.1026, valor bajo y deseable para estudios de clima; relación señal-ruido (valor obtenido de la relación entre la señal y factores de disturbio y se refiere a la capacidad de una cronología para derivar información climática en relación a otros factores no atribuidos a la influencia del clima) de 6.961. Los valores de los parámetros dendrocronológicos indican un adecuado potencial de la especie para reconstrucciones dendroclimáticas (Delgado, 2000; Grissino-Mayer, 2001).

\section{Respuesta climática}

Precipitación. La comparación entre las cronologías EW, LW y RW de $P$. oocarpa en sus versiones estándar y residual del BLP y datos de precipitación acumulada mensual y de temperatura (mínima, máxima, promedio) de las estaciones climáticas Salitre, La Vega, Presa Hurtado y Antonio Escobedo, indican que la mejor respuesta se obtuvo entre la cronología EW versión residual con la precipitación acumulada del período de octubre del año previo a mayo del año actual de crecimiento. Al hacer un promedio de la precipitación acumulada octubre-mayo de las estaciones Presa Hurtado y Antonio Escobedo y analizar su asociación con la cronología residual EW, se obtuvo una correlación de 0.775 ( $\mathrm{p}<0.0000, \mathrm{n}=46,1958-2003)$; es decir, el crecimiento de la madera temprana explica 60\% (valor obtenido de elevar al cuadrado el valor de la correlación) de la variación en precipitación captada por dichas estaciones (Fig. 4).

Temperatura. La relación entre la cronología EW y temperatura promedio mínima, máxima y media mensual de las estaciones climáticas La Vega, El Salitre y Presa Hurtado sólo fue significativa para la temperatura máxima estacional enero-junio $(\mathrm{r}$ $=-0.622, \mathrm{p}<0.0000, \mathrm{n}=46,1956-2001)$, donde la serie de madera temprana explica $38.7 \%$ de la variación estacional en temperatura máxima; esta relación fue negativa; es decir, una mayor temperatura máxima ocasionó menor valor de los índices y viceversa (Fig. 5). 


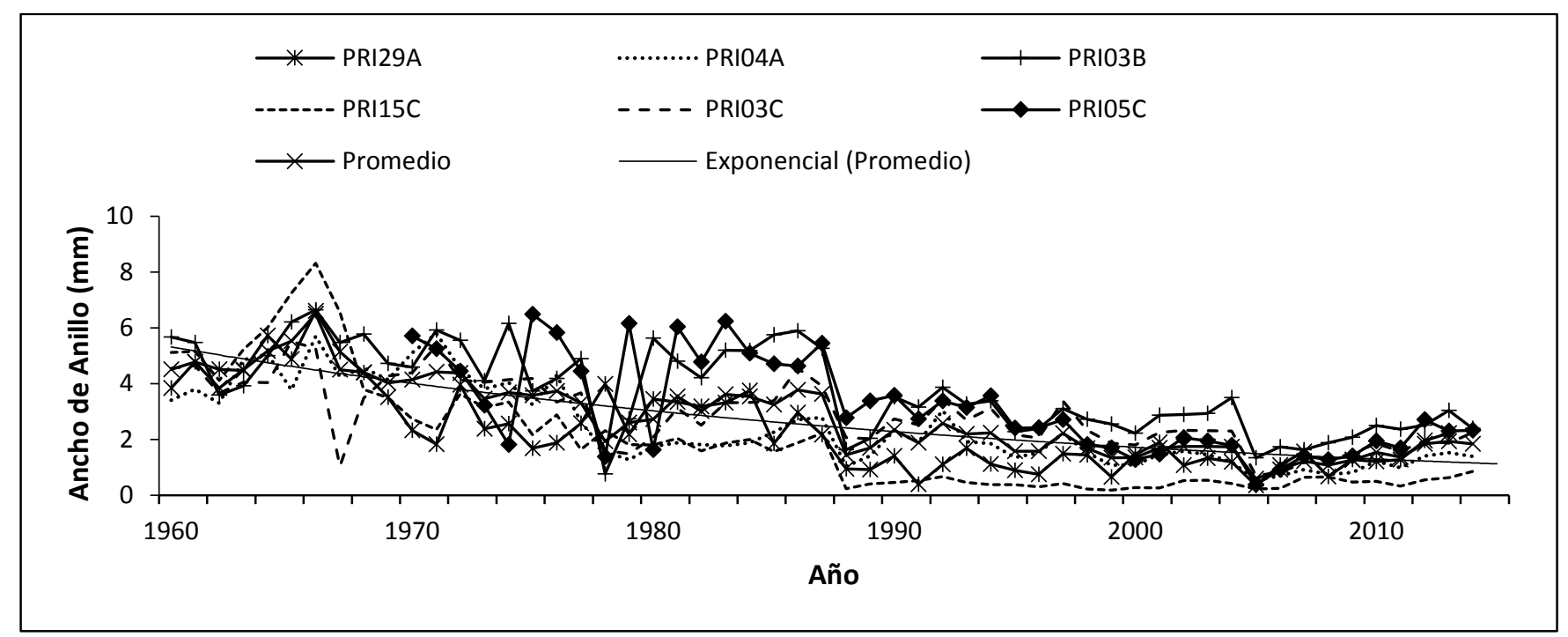

FIGURA 2. Crecimiento radial anual (grosor de anillo) para algunas series de árboles establecidos en la década de 1960 . El crecimiento de las series se ajusta a una curva exponencial negativa (línea continua obscura).

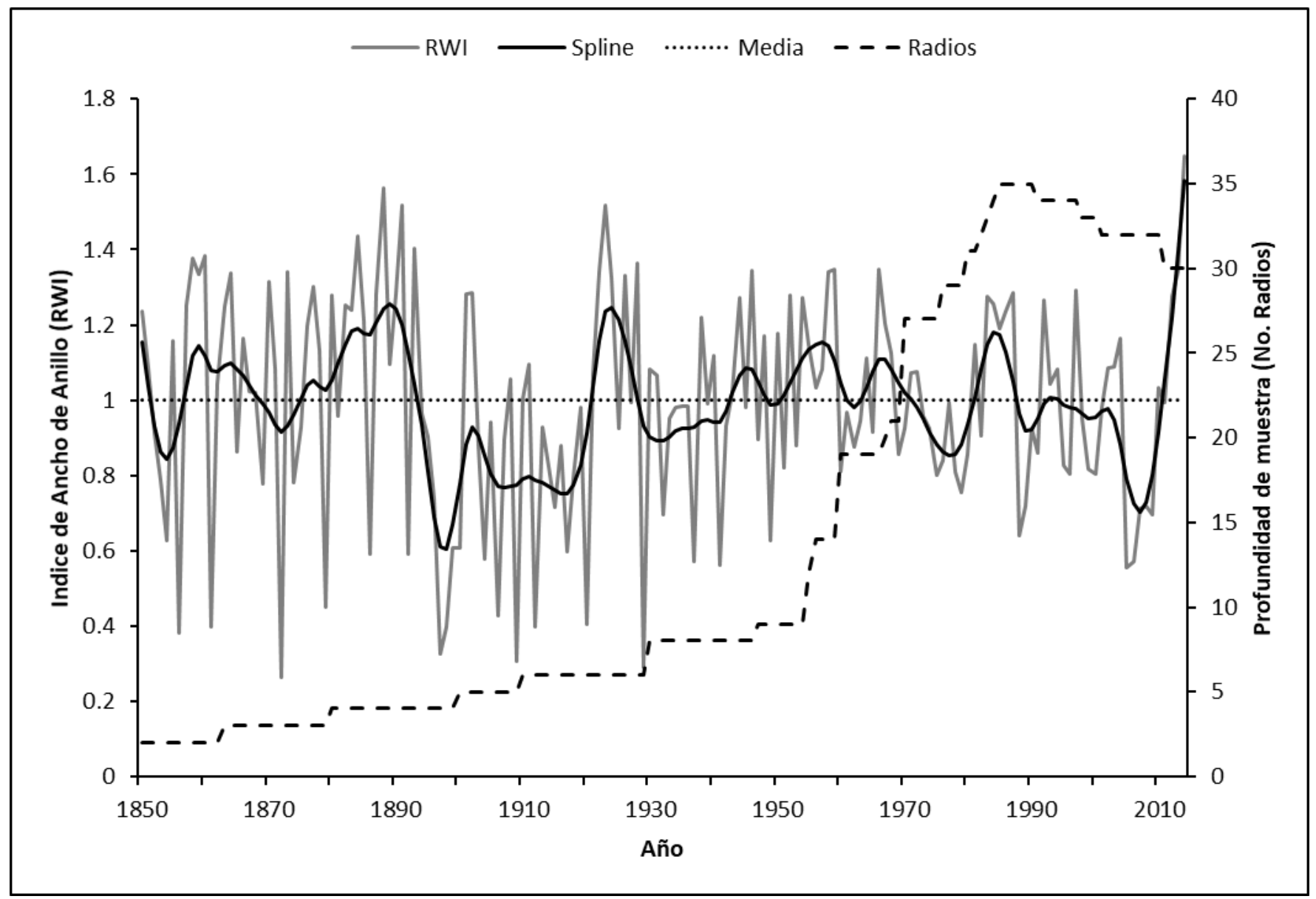

FIGURA 3. Cronología estándar de anillo total de P. oocarpa para el BLP. La línea gris corresponde a los valores anuales de la cronología; la línea horizontal punteada es la media de la cronología; la obscura es una línea flexible para enfatizar eventos de baja frecuencia a nivel decenal y la línea quebrada es el tamaño de muestra (núcleos de crecimiento, radios) que intervinieron para determinar el valor promedio del índice de ancho de anillo. 


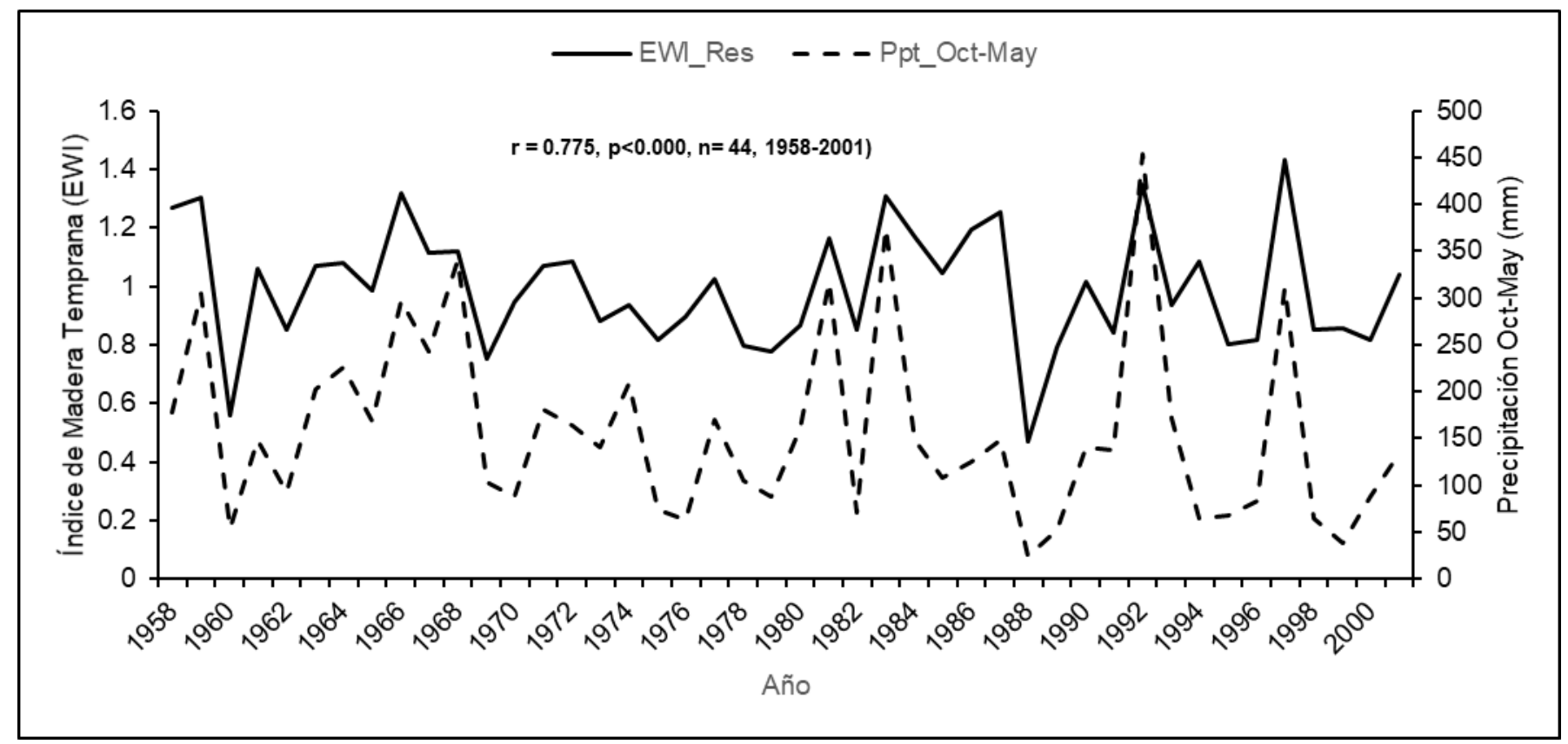

FIGURA 4. Relación entre la cronología de madera temprana, versión residual y datos acumulados promedio de precipitación octubremayo de las estaciones climáticas Presa Hurtado y Antonio Escobedo

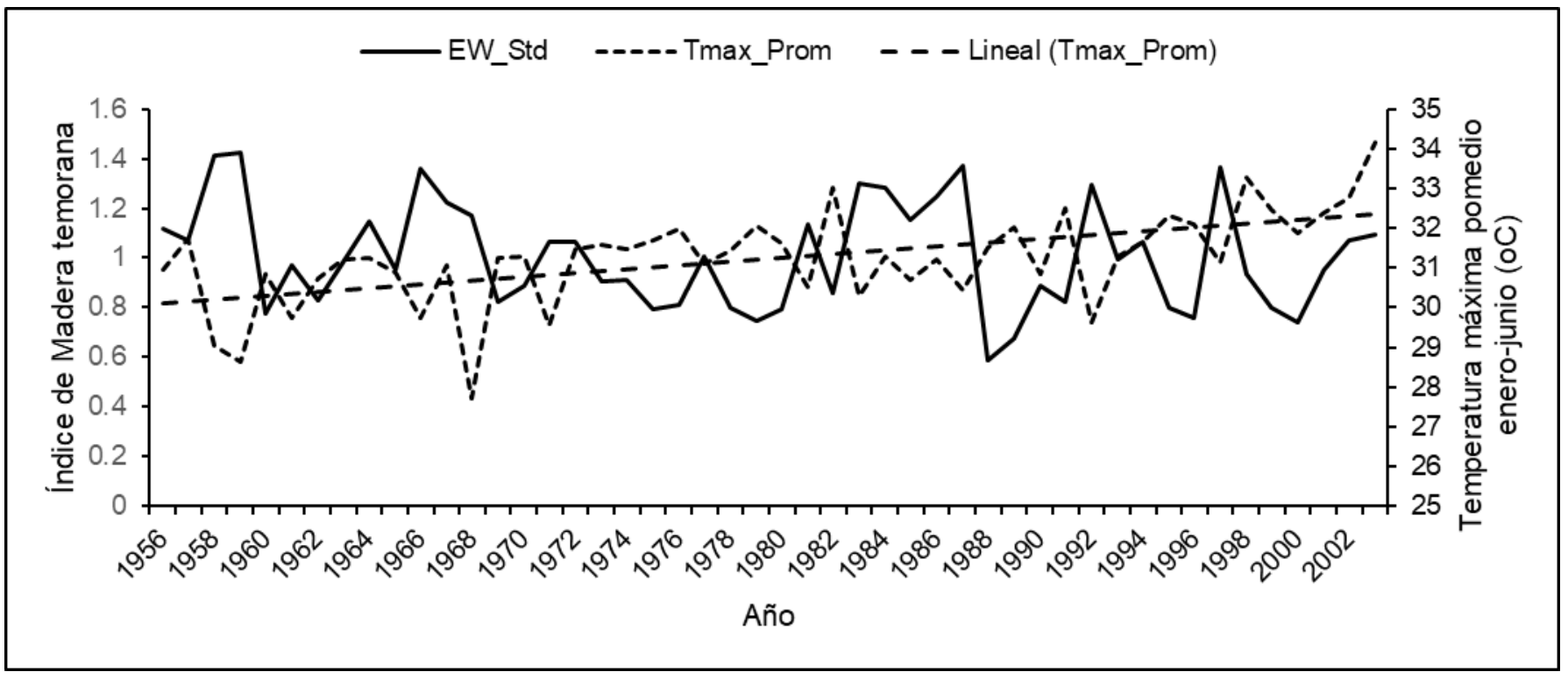

FIGURA 5. Relación entre el índice de crecimiento de las series de crecimiento estándar de la madera temprana y la temperatura máxima promedio estacional del período enero-junio. La línea horizontal seccionada corresponde a una recta de regresión positiva ajustada a los datos de temperatura.

\section{Registros hidrométricos}

La relación entre los índices dendrocronológicos y los registros hidrométricos no indicó significancia para ninguna de las estaciones hidrométricas analizadas. La mayor asociación se determinó entre los índices EW versión estándar y los volúmenes acumulados septiembre-octubre de la estación hidrométrica San Cristobal II, donde la correlación solo alcanzó un valor de 0.175 $(\mathrm{p}=0.22, \mathrm{n}=52,1960-2011)$.

La serie dendrocronológica también se comparó con la diferencia en cota de los niveles registrados del lago de Chapala, 
que consisten en la diferencia entre el valor mínimo y máximo de cota registrados para un año determinado. La asociación observada fue de 0.26 ( $\mathrm{p}=0.055, \mathrm{n}=58,1955-2012)$.

\section{Reconstrucción de precipitación}

Los registros de precipitación se dividieron en dos subperíodos con fines de calibración y verificación; el primer subperíodo cubrió los años de 1958 a 1979 ( $\mathrm{n}=22$ ) y, al compararlo con la serie de EW, tuvo un valor de correlación de 0.811 ( $\mathrm{p}<0.0000$ ); mientras que el segundo sub-período fue de 1980 a $2001(\mathrm{n}=22)$ con un valor de correlación de $0.766(\mathrm{p}<$ 0.000) (Tabla 2).

Las pruebas de calibración y verificación indican que ambos sub-períodos pasaron las diversas pruebas estadísticas involucradas en los análisis y se consideran estadísticamente robustas con fines de reconstrucción (Tabla 3).

Debido a que ambos subperíodos fueron significativos, se utilizó el período total de registros de precipitación disponible (1958-2001) para desarrollar un análisis de regresión y generar el modelo de regresión lineal con fines de reconstrucción. La correlación obtenida del período 1958-2001 fue de 0.775 ( $\mathrm{p}<$ 0.0000); es decir, los crecimientos anuales explican 60\% de la precipitación estacional octubre-mayo, que cubre parte del período otoño-primavera. El análisis de regresión indicó una probabilidad altamente significativa entre ambas variables, por lo que el modelo de regresión lineal utilizado con fines de reconstrucción fue el siguiente:

$$
Y_{t}=-205.6+365.8 X_{i}
$$

Donde:

$\mathrm{Y}_{\mathrm{t}}=$ Precipitación acumulada octubre-mayo $(\mathrm{mm})$

$\mathrm{X}_{\mathrm{i}}=$ Índice de madera temprana de la versión residual de la cronología

$\mathrm{Al}$ aplicar el modelo lineal en la longitud total de la serie de madera temprana, se generó una reconstrucción de precipitación estacional, que cubre los últimos 165 años y que indica alta variabilidad interanual y multianual (Fig. 6).

TABLA 2. Períodos de calibración y estadísticos de la regresión entre los registros de precipitación y los índices de la cronología residual de madera temprana.

\begin{tabular}{|c|c|c|c|c|c|c|c|c|}
\hline \multirow[t]{3}{*}{ Período } & \multirow[t]{3}{*}{$\mathrm{R}^{2} \mathrm{Adj} \mathrm{j}^{1}$} & \multicolumn{2}{|c|}{ Coeficiente (mm) } & \multicolumn{2}{|c|}{ Error estándar (mm) } & \multirow{2}{*}{\multicolumn{2}{|c|}{$\begin{array}{l}\text { Estadístico-t } \\
\left(\mathrm{H}_{\circ}: B=\mathrm{O}\right)\end{array}$}} & \multirow[t]{3}{*}{ Regresión (prob.) } \\
\hline & & & & & & & & \\
\hline & & Bo & $B_{1}$ & $B_{0}$ & $B_{1}$ & $B o$ & $B_{1}$ & \\
\hline 1958-1979 & 0.64 & -177.9 & 348.2 & 56.3 & 56.1 & $-3.16^{* *}$ & $6.21^{* *}$ & $4.5618 \mathrm{E}-06^{* *}$ \\
\hline $1980-2001$ & 0.57 & -230.2 & 380.1 & 73.0 & 71.34 & $-3.15^{* *}$ & $5.33^{* *}$ & $3.2448 \mathrm{E}-05^{* *}$ \\
\hline $1958-2001$ & 0.59 & -205.6 & 365.8 & 47.0 & 45.97 & $-4.41^{* *}$ & $7.95^{* *}$ & $6.4137 \mathrm{E}-10^{* *}$ \\
\hline
\end{tabular}

*Significativo, p<O.05; **Significativo, $\mathrm{p}<0.01$; ' $\mathrm{R}^{2}$ ajustada acorde a grados de libertad (Draper y Smith, 1981)

TABLA 3. Estadísticos de verificación entre los datos de precipitación observados y reconstruidos.

\begin{tabular}{|c|c|c|c|c|c|}
\hline Periodo & $\begin{array}{l}\text { Correlación } \\
\text { de Pearson }\end{array}$ & $\begin{array}{l}\text { Reducción del } \\
\text { error }^{2}\end{array}$ & Valor de " $t$ "3 & Prueba de signos ${ }^{4}$ & $\begin{array}{l}\text { Primera } \\
\text { negativa }{ }^{5}\end{array}$ \\
\hline 1958-1979 & $0.82^{*}$ & $0.66^{*}$ & $4.09^{*}$ & $4^{*}$ & $3^{*}$ \\
\hline $1980-2001$ & $0.78^{*}$ & $0.59^{*}$ & $3.1^{*}$ & 9 NS & $4^{*}$ \\
\hline
\end{tabular}

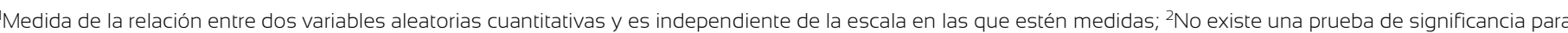

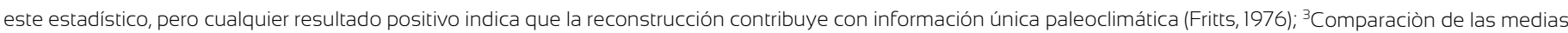

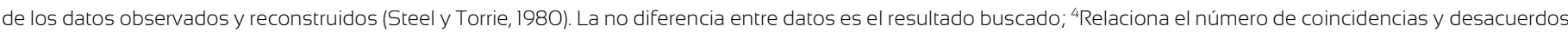

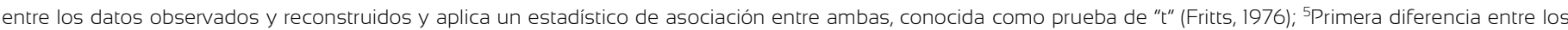

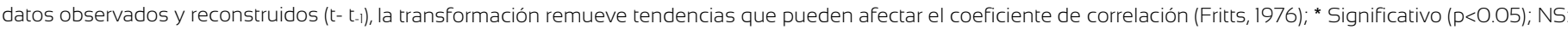
No significativo 


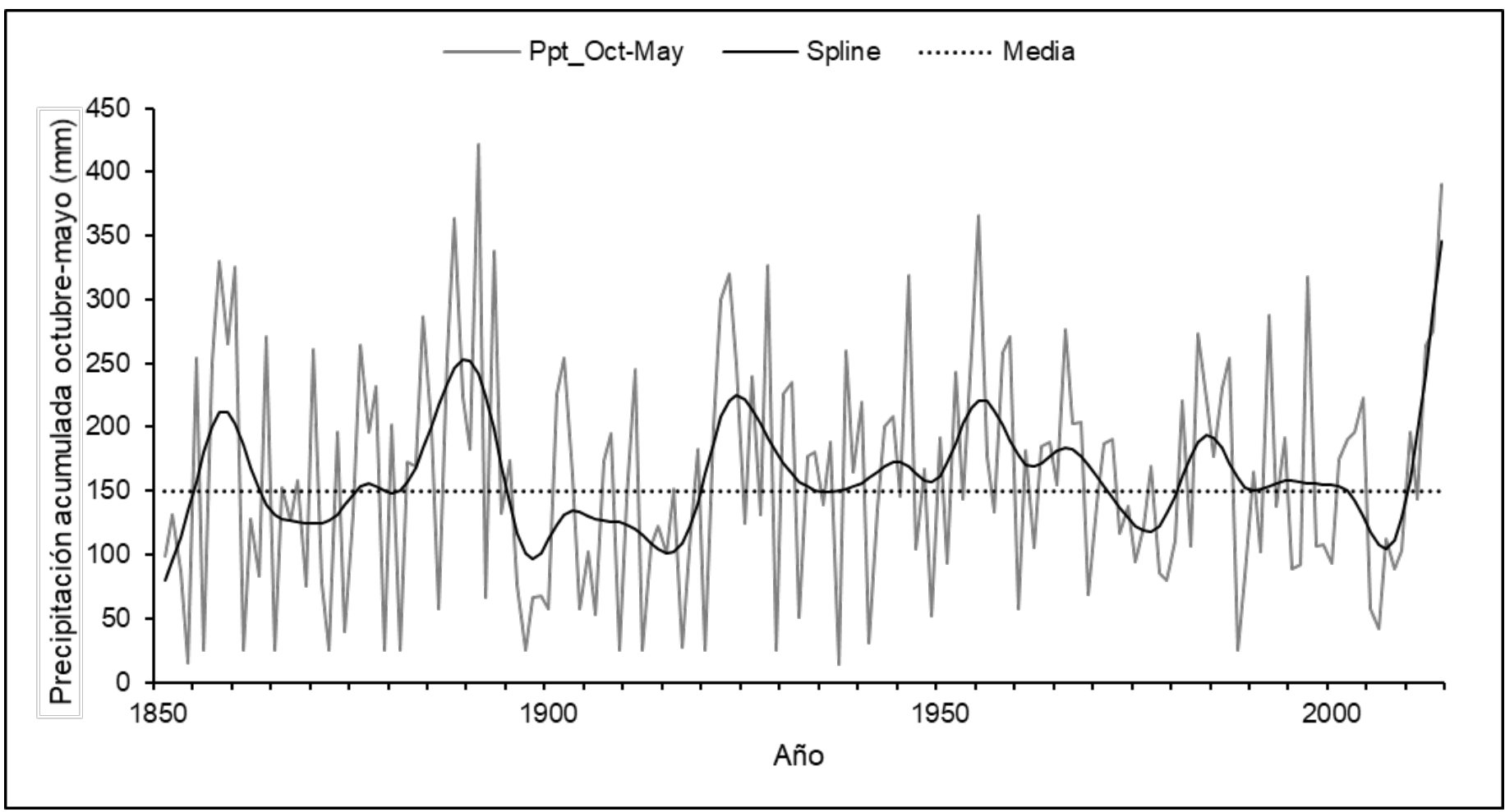

FIGURA 6. Reconstrucción de precipitación estacional otoño-primavera (octubre-mayo) para el área de protección de flora y fauna Bosque La Primavera, Jalisco. La línea sólida en gris son los valores anuales; la línea horizontal punteada corresponde a la media de los datos registrados octubre-mayo y la línea obscura es una línea flexible (spline) a nivel década para resaltar eventos de baja frecuencia (multianuales).

Las sequías fueron un fenómeno recurrente en esta reconstrucción y un $44.5 \%$ de los años mostraron precipitación inferior a la media registrada $(150.0 \mathrm{~mm})$, donde la media de estos años secos fue de $83.8 \mathrm{~mm}$, con una desviación estándar de $40.0 \mathrm{~mm}$. Períodos con varios años consecutivos de sequía se presentaron de 1851 a 1856, 1861 a 1865, 1871 a 1875, 1896-1900, 1904 a 1906, 1909 a 1918, 1915 a 1920, 1945 a 1959, 1970 a 1982, 1995 a 2000 y 2005 a 2009. Por otra parte, los períodos húmedos ocurrieron de 1857 a 1860, 1876 a 1878, 1882 a 1885, 1887 a 1991 , 1901 a 1903, 1921 a 1924, 1930 a 1931, 1938 a 1940, 1943 a 1944, 1958 a 1959, 1963 a 1968, 1971 a 1972, 1983 a 1987, 2001 a 2004 y 2012 a 2014.

\section{Influencia de fenómenos circulatorios}

La relación entre los índices de las diferentes bandas de crecimiento (EW, LW, RW) de P. oocarpa y los diversos índices de fenómenos atmosféricos de circulación general mostraron correlaciones muy bajas y no significativas (Tabla 4).

No obstante, la ausencia de una correlación significativa entre los diferentes fenómenos circulatorios; el fenómeno ENSO tanto en su fase Niño o Niña, si afectó la precipitación en el BLP, particularmente para eventos considerados como moderados a intensos, con una influencia favorable en la precipitación durante la fase cálida del Niño e inferior en la fase La Niña (Tabla 5). 
TABLA 4. Relación entre la serie dendrocronológica de P. oocarpa en BLP y diversos índices de fenómenos circulatorios.

\begin{tabular}{lllllll}
\hline $\begin{array}{l}\text { Fenómeno } \\
\text { circulatorio }\end{array}$ & I'́ndice & $\begin{array}{l}\text { 2Tipo de } \\
\text { Cronología }\end{array}$ & Período (años) & $\begin{array}{l}\text { Período } \\
\text { estacional }\end{array}$ & ${ }^{3} r$ & 4Prob. (p-value) \\
\hline ENSO & SOI & RWI_Std & $1878-2013$ & Jul-Dic & 0.145 & 0.09 \\
& MEI & RWI_Std & $1871-2005$ & Jun-Dic & 0.153 & 0.07 \\
& TRI & RWI_Std & $1894-1995$ & Enero & 0.140 & 0.15 \\
PDO & PDO & RWI_Std & $1900-2010$ & Ene-Ago & 0.100 & 0.28 \\
AMO & AMO & RWI-Res & $1948-2014$ & Sep-Dic & 0.090 & 0.45 \\
PDSI & PDSI & EWI_Res & $1950-2008$ & Junio & 0.234 & 0.07
\end{tabular}

'Allan SOI invernal (Dic-Feb): índices de SOI basados en la diferencia de presión de la temperatura de agua del mar entre las islas de Tahiti y Darwin, Australia (Allan et al. 1996; NOAA, 2014); MEl: Índice Multivariado de ENSO, que se fundamenta en variables registradas en el Pacífico Tropical, las cuales son presión al nivel del mar (P), componentes del viento superficial tanto zonal (U) como meridional (V), temperatura del agua superficial del mar (S), temperatura del aire(A) y fracción total de nubosidad del cielo (C) (Wolter y Timlin, 2Ol1); TRl: Índice de Lluvia Tropical, constituye una estimación de la variabilidad de ENSO, al fundamentarse en las anomalías de precipitación en la región del Pacífico central, lo que le da una mayor estabilidad que el índice Tahiti-Darwin (Wright, 1979); PDO: El PDO consiste en un patrón de variabilidad climática del Pacífico que cambia a escala interdecadal en períodos aproximados de 20 años a 30 años (Hare y Mantua, 2000); AMO: corriente oceánica que se considera afecta la temperatura de la superficie del agua del mar en la porción norte del Océano Atlántico en escalas de tiempo multidecadales (Van Oldenborgh et al. 2009); El PDSI es un índice de balance de humedad que se basa en registros de precipitación y de temperatura de estaciones climáticas y constituye un "proxy" razonable del crecimiento del árbol (Cook et al. 1999). Los valores de PDSI son los reconstruidos para el centro de México (Stahle et al. 2011).

2RWI_Std: cronología de anillo total versión estándar, EWI_Res cronología de madera temprana versión residual

${ }^{3}$ r: correlación

4Prob. (p-value): valor de significancia

TABLA 5. Relación entre la intensidad de las diversas fases cálida (Niño) y fría (Niña) de ENSO y la precipitación reconstruida en el BLP.

\begin{tabular}{llllll}
\hline Débil & $\begin{array}{c}\text { El Niñol } \\
\text { Moderado }\end{array}$ & Intenso & Débil & La Niña \\
\hline $1969(68.9)$ & $1986(231.1)$ & $1958(258.2)$ & $1951(92.7)$ & $1973(116.6)$ & $1975(93.9)$ \\
$1976(112.4)$ & $1987(253.7)$ & $1966(276.5)$ & $1955(365.5)$ & $1998(106.8)$ & $1988(25.0)$ \\
$1995(88.4)$ & $1991(102.6)$ & $1972(190.6)$ & $1964(188.7)$ & $1999(108.2)$ & $1989(83.9)$ \\
$2005(57.0)$ & $2002(190.2)$ & $1983(273.4)$ & $1967(202.2)$ & $2000(93.6)$ & \\
$2006(42.1)$ & $2003(195.5)$ & $1997(318.3)$ & $1970(139.9)$ & $2007(112.6)$ & \\
& $2009(103.3)$ & & $1971(186.6)$ & $2008(88.8)$ & \\
& $2010(196.3)$ & & $1974(137.5)$ & & \\
75.7 & & & $1984(221.6)$ & & \\
& 181.8 & 263.4 & 186.5 & 104.5 & 67.6 \\
\hline
\end{tabular}

'Eventos El Niño (fase cálida), La Niña (fase fría) en el pacífico tropical para la región 3.4 (5 N-5 S, 120-170 W), período 1951-2014. Los eventos se definen como la presencia de 5 meses consecutivos con un traslape de 3 meses, donde la anomalía de temperatura es al menos de $+0.5^{\circ} \mathrm{C}$ para eventos El Niño y por debajo de - $0.5^{\circ} \mathrm{C}$ para La Niña. La intensidad del evento se define en función al rango de valores; así valores entre 0.5 a 0.9 de anomalía de la temperatura del agua del mar corresponde a un Niño débil, entre 1.0 a 1.4 a uno moderado y >a 1.5 a uno intenso (NOAA, 2016).

Los valores entre paréntesis constituyen la precipitación reconstruida octubre-mayo y los valores en negritas es el promedio de los años con la anomalía.

\section{DISCUSIÓN}

El incremento anual de los anillos de crecimiento (incremento radial) de $P$. oocarpa mostró alta variación interanual; un efecto que reduce el ancho de anillos se produce durante años secos, o bien durante el año de incendio o uno dos años después del mismo, debido a una disminución en el tejido fotosintético
(Schweingruber, 1996). Esta situación ocurrió en incendios que impactaron una amplia superficie en el BLP, como los ocurridos durante 1998, 2005 y 2012. En otros casos, su crecimiento puede incrementarse después de un incendio, particularmente cuando este es superficial y de baja intensidad, lo que favorece a una eliminación de la competencia, liberación de nutrientes y mayor 
disponibilidad hídrica (Brown y Swetnam, 1994). Ambas situaciones parecen haber ocurrido en las series de crecimiento de BLP, lo cual probablemente es el resultado de una interacción entre la intensidad del incendio y las condiciones climáticas dominantes para el año del evento, ya que condiciones secas durante la estación de crecimiento, por sí solas, dan lugar a la producción de anillos de tamaño reducido (Fritts, 1976).

El proceso de estandarización que elimina las tendencias biológicas generó una serie de crecimiento muy sensible a variaciones interanuales y multianuales climáticas, lo que se refleja en parámetros dendrocronológicos adecuados con fines dendrocronológicos, como fue la inter-correlación entre series (0.506), sensibilidad media (0.322) y auto-correlación de primer orden de 0.102, que en comparación a otras series dendrocronológicas desarrolladas para la región; estas tuvieron valores menos deseables con fines de reconstrucción como fue el caso de $P$. douglasiana en Sierra de Manantlán, Jalisco, que mostró una inter-correlación entre series de 0.474 , sensibilidad media de 0.201 y auto-correlación de primer orden de 0.57 (CeranoParedes et al., 2013); situación similar ocurrió para series dendrocronológicas con ahuehuete (Taxodium mucronatum) presente en afluentes que drenan al lago de Chapala (Villanueva et al., 2012). Los valores de los parámetros señalados, a pesar de no ser restrictivos para realizar o no una reconstrucción de variables climáticas, sí son indicativos del potencial que puede tener una especie como $P$. oocarpa para el desarrollo de reconstrucciones dendroclimáticas.

La respuesta climática observada para la especie fue similar a la determinada en otras coníferas, cuyo crecimiento anual responde a la precipitación invierno-primavera, como es el caso de P. douglasiana y P. maximinoi (Cerano et al., 2013; Rangel, 2015) distribuidas en la parte occidental del estado de Jalisco y Pseudotsuga menziesii y otras especies de pino en el norte y noreste de México (Villanueva, Fulé, Cerano, Estrada y Sánchez, 2009).

La relación positiva entre crecimiento y precipitación acumulada otoño-primavera, contrasta con el efecto negativo originado por un incremento en la temperatura máxima promedio de los meses enero-mayo; situación que probablemente acelera la evaporación del agua almacenada en el suelo y restringe el crecimiento de la madera temprana (Fritts, 1976).
El incremento de la temperatura máxima en el BLP que pasó de $31.7{ }^{\circ} \mathrm{C}$ a $32.2^{\circ} \mathrm{C}\left(+1.5^{\circ} \mathrm{C}\right)$ en el período de 1956 a 2001, parece impactar negativamente el incremento radial de la especie. Esta tendencia en el incremento de la temperatura probablemente acelerada por actividades industriales y uso de vehículos motorizados en la urbe metropolitana de Guadalajara, con una población actual de 4.8 millones de habitantes, contribuyen al calentamiento global, lo que puede tener consecuencias negativas a corto plazo, como los señalan modelos de predicción climática para México, que predicen una tendencia a sequías más frecuentes e intensas en las siguientes décadas (Seager et al., 2009; Feng y Fu, 2013).

Los períodos secos observados en la reconstrucción como los ocurridos de 1851 a 1856, 1896 a 1900, 1909 a 1925, 1945 a 1959, 1995 a 2000 y 2005 a 2009, se encuentran presentes en reconstrucciones desarrolladas para la parte sur-occidental del estado de Jalisco (Villanueva et al., 2012; Cerano et al., 2013; Rangel, 2015; Stahle et al., 2016) y algunas como las de las décadas de 1860, 1890 y 1950 en el centro, norte y noreste de México (Cleaveland, Stahle, Therrell, Villanueva y Burns, 2003, Villanueva-Díaz et al., 2007; Stahle et al., 2011); situación que indica que estas sequías fueron originadas por patrones de circulación atmosférica de gran alcance como es ENSO (Allan, Lindesay y Parker, 1996; Stahle et al., 1998).

Durante el siglo XIX, las sequías más intensas para el occidente de México ocurrieron entre 1884 y 1886 y en el período 1892-1896 (Contreras, 2005) y tuvieron un alto impacto en la disponibilidad de alimentos (Florescano, 1995). Esta sequía se extendió en el BLP de 1892 a 1900, excepto 1893. Durante el siglo XX las sequías más sobresalientes se presentaron en los períodos 1948-1954, 1960-1964, 1970-1978 y 1993-1996. En la reconstrucción, los años más secos fueron 1949, 1955, 1960 , 1962, 1969, 1970, 1975, 1978, 1998 y 2000. En el presente siglo el período de 2005 a 2007 fue crítico en BLP, particularmente debido a la presencia de incendios de alta intensidad que afectaron una gran superficie, como fue el caso del ocurrido en 2005, que daño 8702 ha (Huerta y Ibarra, 2014).

Los períodos húmedos son de gran importancia económica, aunque, en general, se les presta una menor atención en comparación con las sequías, las cuales en términos sociales y económicos infringen mayor daño a la sociedad. En este estudio 
fueron evidentes los años húmedos de 1855-1860, 1888-1890, 1922-1924, 1954-1956, 1992, 1997 y 2014, muchos de los cuales coinciden con una recuperación sustancial en los niveles del lago de Chapala (Villanueva et al., 2012). No obstante, es pertinente señalar que, en los últimos 50 años, los volúmenes que alcanzan al lago de Chapala a través de sus tributarios han sido modificados para satisfacer una mayor demanda con fines de irrigación y para otros usos, lo cual distorsiona los volúmenes reales de escurrimiento que deberían almacenarse en el lago.

La influencia de fenómenos circulatorios como ENSO en la precipitación de BLP no fue tan notoria como en sitios de la Sierra Madre Occidental de los estados de Chihuahua y Durango, donde este fenómeno puede explicar más de $40 \%$ de la precipitación invierno-primavera (Stahle et al., 1998; Villanueva et al., 2014; Díaz-Ramírez, Villanueva-Díaz y Cerano-Paredes, 2016; Stahle et al., 2016). No obstante, se determinó que eventos de moderados a intensos de la fase cálida de ENSO incrementan la precipitación otoño-primavera, mientras que la fase fría la reduce, excepto en eventos débiles del Niño y la Niña, que muestran un impacto similar en términos de producir un decremento en la precipitación; situación que ha sido reportada anteriormente para el centro de México, donde la fase Niña favorece un incremento en la precipitación, comparado con el norte de México, donde la reduce (Magaña, Pérez, Vázquez, Carrizosa y Pérez, 1999; Stahle et al., 2016).

Otros fenómenos circulatorios como el PDO no mostraron un efecto significativo en la precipitación observada en esta región y al parecer su impacto se circunscribe más en la parte noroeste de México, en particular la península de Baja California (Biondi, Gerhunov y Cayan, 2001; Villanueva et al., 2015); mientras que AMO y PDO tienen más influencia en la península de Yucatán (Méndez y Magaña, 2010); probablemente tormentas tropicales o lluvias convectivas producidas por el fenómeno del Monzón de Norteamérica pudieran tener un mayor impacto, pero se requiere un análisis más detallado del mismo.

\section{CONCLUSIONES}

Una cronología de Pinus oocarpa de madera temprana, tardía y de anillo total de 165 años (1850-2014) desarrollada para el BLP mostró parámetros dendrocronológicos deseables con fines de reconstrucción e incluso superiores a las series de crecimiento de otras coníferas para el sur de Jalisco, con las que existen reconstrucciones dendroclimáticas previas.

La respuesta entre la serie de crecimiento de $P$. oocarpa, los registros de precipitación y la temperatura de estaciones climáticas aledañas al BLP señaló una respuesta significativa de la precipitación acumulada octubre-mayo y una relación significativa pero negativa con la temperatura máxima promedio enero-junio. La reconstrucción de precipitación estacional muestra alta variabilidad interanual y multianual y capta con fidelidad algunos de los períodos más secos que se presentaron a nivel nacional, como son las sequías de las décadas de 1860, 1890, 1950 , pero también detecta algunas de las sequías que afectaron el occidente de México como las de los períodos 1884-1886, 1892-1896, 1948-1954, 1960-1964, 1970-1978 y 1993-1996.

Durante el período de registros disponibles, 1956-2001, la temperatura máxima se incrementó de $31.7^{\circ} \mathrm{C}$ a $32.2^{\circ} \mathrm{C}(+1.5$ ${ }^{\circ} \mathrm{C}$ ); este incremento puede tener la influencia de acciones antropogénicas, ya que el BLP se encuentra aledaño a la urbe de Guadalajara, con una población cercana a los cinco millones de habitantes. De continuar esta tendencia, el balance hidrológico se verá afectado, al incrementarse la evaporación y, por ende, una menor disponibilidad hídrica para coníferas y otras latifoliadas que habitan suelos someros, ácidos, de baja capacidad de almacenamiento hídrico y de pobre calidad de sitio.

La asociación entre las series dendrocronológicas de $P$. oocarpa y los diversos índices de fenómenos de circulación general no fue significativa; sin embargo, para eventos específicos de ENSO, la precipitación en el BLP fue superior en eventos moderados a cálidos (El Niño) y disminuyó durante eventos fríos (La Niña) y fue inferior a la media registrada tanto en eventos débiles Niño o Niña.

Dada la incidencia de incendios, particularmente inducidos en el BLP, aunado a una temperatura máxima en aumento, probablemente acelerado por acciones antropogénicas, se deben considerar estas variables para determinar las acciones de manejo que permitan la conservación de las especies arbóreas en este ecosistema, de fundamental importancia para el área metropolitana de Guadalajara. 


\section{RECONOCIMIENTOS}

La presente investigación fue apoyada con fondos de los proyectos fiscales Inifap "Influencia de patrones atmosféricos circulatorios en la frecuencia de incendios forestales en la Sierra Madre Occidental" y el de "Reconstrucción de caudales e influencia de fenómenos de circulación general en cuencas del Pacífico Mexicano”.

\section{REFERENCIAS}

Allan, R., Lindesay J., \& Parker, D. (1996). El Niño/Southern Oscillation y climate variability. Collingwood, Australia: Atmospheric Research, Australian National Univesity.

Banco Nacional de Datos de Aguas Superficiales [Bandas]. (2006). Banco Nacional de Datos de Aguas Superficiales. Morelos, México: Instituto Mexicano de Tecnología del Agua.

Baron, J. S., Gunderson L., Allen, C. D., Fleishman, E., McKenzie, D., Meyerson, L. A., Oropeza, J., \& Stephenson, N. (2009). Options for national parks and reserves for adapting to climate change. Environmental Management, 44,1033-1042. doi: 10.1007/s00267009-9296-6

Biondi, F., Gershunov, A., \& Cayan. D. R. (2001). North pacific decadal climate variability since 1661. Journal of Climate, 14(1), 5-10. doi: 10.1175/1520-0442(2001)014\%3C0005:NPDCVS\%3E2.0.CO;2

Brown, P. M., \& Swetnam, T. W. (1994). A cross-dated fire history from coast redwood near Redwood National Park, California. Canadian Journal of Forest Research, 24, 21-31.

Cerano-Paredes, J., Méndez-González, J., Amaro-Sánchez, A., Villanueva-Díaz, J., Cervantes-Martínez, R., \& Rubio-Camacho, E. (2013). Reconstrucción de precipitación invierno-primavera con anillos anuales de Pinus douglasiana en la Reserva de la Biósfera de Manantlán, Jalisco. Revista Chapingo Serie Ciencias Forestales y del Ambiente, XIX(3), 413-423. doi: 10.5154/r.rchscfa.2013.02.007

Cleaveland, M. K., Stahle, D. W., Therrell, M. D., Villanueva D. J., \& Burns, B. T. (2003). Tree-ring reconstructed precipitation and tropical teleconnections in Durango, Mexico. Climatic Change, 59, 369-388. doi: 10.1023/A:1024835630188

Comisión Nacional de Áreas Naturales Protegidas [Conanp]. (2000). Programa de manejo área de protección de flora y fauna La Primavera. Ciudad de México, México: Secretaría de Medio Ambiente y Recursos Naturales.

Contreras S. C. (2005). Las sequías en México durante el siglo XIX. Investigaciones Geográficas, Boletín del Instituto de Geografía, 56,118-133.

Cook, E. R. (1987). The decomposition of tree-ring series for environmental studies. Tree-Ring Bulletin, 47, 37-59.

Cook, E. R., Meko, D. M., Stahle, D. W., \& Cleaveland, M. K. (1999). Drought reconstruction for the continental United States. Journal of Climate, 12, 1145-1162. doi: 10.1175/15200442(1999)012<1145:DRFTCU>2.0.CO;2
Delgado, C. S. (2000). Aplicaciones estadísticas en análisis dendrocronológicos. En F. A. Roig (Compilador), Dendrocronología en América Latina (79-102). Mendoza, Argentina: EDIUNC.

Díaz-Ramírez, B., Villanueva-Díaz, J., \& Cerano-Paredes, J. (2016). Reconstrucción de la precipitación estacional con anillos de crecimiento para la región hidrológica Presidio-San Pedro. Madera y Bosques, 22(1), 111-123. doi: 10.21829/myb.2016.221480

Dodds, W. K., Perkin, J. S., \& Gerken, J. E. (2013). Human impact on freshwater ecosystem services: a global perspective. Environmental Science and Technology, 47(16), 9061-9068.

Draper, N. R., \& Smith, H. (1998). Applied regression analysis (3a ed.). Nueva York, Estados Unidos: Wiley.

ERIC III, versión 3.2. (2013). Jiutepec, Morelos, México. Instituto Mexicano de Tecnología del Agua.

Feng, S., \& Fu, Q. (2013). Expansion of global drylands under a warming climate. Atmospheric Chemistry and Physics, 13, 10081-10094.

Florescano, E., \& Swan, S. (1995). Breve historia de la sequía en México. Veracruz, México: Universidad Veracruzana.

Fritts, H. C. (1976). Tree rings and climate. Nueva York, Estado Unidos: Academic Press.

Fritts, H. C. (1991). Reconstructing large-scale climatic patterns from tree-ring data: a diagnostic analysis. Tucson, Estados Unidos: The University of Arizona Press.

Gallegos R., A., González C., G. A., Cabrera O., R. G., Marcelli S., C., \& Hernández A., E. (2014). Efecto de la recurrencia de incendios forestales en la diversidad arbórea. Revista Mexicana de Ciencias Forestales, 5(24), 110-125.

García, E. (2004). Modificaciones al sistema de clasificación climática de Köppen. Ciudad de México, México: Instituto de Geografía, UNAM.

Grissino-Mayer, H. D. (2001). Evaluating crossdating accuracy: a manual and tutorial for the computer program COFECHA. Tree Ring Research, 57(2), 205-221.

Hare, S. R., \& Mantua, N. J. (2000). Empirical evidence of North Pacific regime shifts in 1977 and 1989. Progress in Oceanography, 47(2-4), 103-145. doi: 10.1016/S0079-6611(00)00033-1

Holmes, R. L. (1983). Computer-assisted quality control in tree-ring dating and measurement. Tree-Ring Bulletin, 43, 69-78.

Huerta, F. M., \& Ibarra, J. L. (2014). Incendios en el bosque La Primavera (Jalisco, México): un acercamiento a sus posibles causas y consecuencias. Ciencia UAT, 9(1), 23-32.

Instituto Nacional de Estadística y Geografía [Inegi]. (2015). Censo de población y vivienda 2015 - Estados Unidos Mexicanos resultados definitivos por entidad y municipio. México, Ciudad de México.

Magaña, V., Pérez, J. L., Vázquez, L. L., Carrizosa, E., \& Pérez, J. J. (1999). El Niño y el clima. En V. Magaña (Ed.), Los impactos del Niño en México (pp. 23-68). Ciudad de México, México: SEPCONACYT. 
Méndez, M., \& Magaña, V. (2010). Regional aspects of prolonged meteorological droughts over Mexico and Central America. Journal of Climate, 23,1175-1188. doi: 10.1175/2009JCLI3080.1

Administración Nacional Oceánica y Atmosférica [NOAA]. (2014). Southern Oscillation Index (SOI). Recuperado de http: //www.ncdc.noaa.gov/teleconnections/enso/indicators/soi.ph p.

Administración Nacional Oceánica y Atmosférica [NOAA]. (2016). El Niño and La Niña years and intensities base on Oceanic Niño Index (ONI). Recuperado de http://ggweather.com/enso/oni.htm.

Panshin, A. J., \& De Zeeuw, C. (1980). Texbook of Wood technology: structure, identification properties, and uses of the commercial Woods of the United States and Canada. Nueva York, Estados Unidos: McGraw-Hill series in forest resources V.1.

Rangel H., C. (2015). Variabilidad de la precipitación estacional reconstruida otoño-invierno para el sur de Jalisco con series dendrocronológicas (Tesis de licenciatura no publicada). URUZA, Bermejllo, Durango, México.

Schweingruber, F. H. (1996). Tree rings and environment dendroecology. Berne, Suiza: Swiss Federal Institute for Forest, Snow and Landscape Research, WSL/FNP, Birmensdorf-Berne.

Seager, R., Ting, M., Davis, M., Cane, M., Nike, N., Nakumara, J., Lie, C., Cook, E., \& Stahle, D.W. (2009). Mexican drought: an observational modeling and tree ring study of variability and climate change. Atmósfera, 22(1), 1-31.

Stahle, D. W., D’Arrigo, R. D., Krusic, P. J., Cleaveland, M. K., Cook, E. R., Allan, R. J., Cole, J. E., Dunbar, R. B., Therrell, M. D., Guy, D. A., Moore, M. D., Stokes, M. A., Burns, B. T., Villanueva-Diaz, J., \& Thompson, L. G. (1998). Experimental dendroclimatic reconstruction of the Southern Oscillation. Bulletin of the American Meteorological Society, 70(10), 2137-2152. doi: 10.1175/15200477(1998)079\%3C2137:EDROTS\%3E2.0.CO;2

Stahle, D. W., Burnette, D. J., Villanueva-Diaz, J., Heim, R. R., Fye, F. K, Cerano-Paredes, J., Acuna-Soto, R., \& Cleaveland, M. K. (2011). Pacific and Atlantic influences in Mesoamerican over the past millennium. Climate Dynamics: doi 10.1007/s00382-011-1205$\mathrm{z}$

Stahle, D. W., Cook, E. R., Burnette, D. J., Villanueva, J., Cerano, J., Burns, J. N., Griffin, D., Cook, B. J., Acuña, R., Torbenson, M. C. A, Sjezner, P., \& Howard, J. M. (2016). The Mexican drought atlas: tree-ring reconstructions of the soil moisture balance during the late pre-Hispanic, colonial, and modern eras. Quaternary Science Review, 149, 34-60. doi: https://doi.org/10.1016/j.quascirev.2016.06.018

Steel, R. G. D., \& Torrie, J. H. (1980). Principles and procedures of statistics: a biometrical approach. Second edition. Nueva York, Estados Unidos: McGraw Hill.

Stokes, M. A., \& Smiley, T. L. (1968). Tree-ring dating. Chicago, Estados Unidos: The University of Chicago Press.
Van Oldenborgh, G. J., te Raa, L. A., Dijkstra, H. A., \& Philip, S. Y. (2009). Frequency- or amplitude-dependent effects of the Atlantic meridional overturning on the tropical Pacific Ocean. Ocean Science, 5 (3), 293-301.doi: 10.5194/os-5-293-2009

Villanueva-Díaz, J, Stahle, D. W., Luckman, B. H., Cerano-Paredes, J., Therrell, M. D., \& Cleaveland, M. K. (2007). Winter-spring precipitation reconstructions from tree rings for northeast Mexico. Climatic Change, 83,117-131. doi 10.1007/s10584-0069144-0

Villanueva D., J., Fulé, P. Z., Cerano P., J., Estrada A., J., \& Sánchez C., I. (2009). Reconstrucción de la precipitación estacional para el barlovento de la Sierra Madre Occidental con anillos de crecimiento de Pseudotsuga menziesii (Mirb.) Franco. Ciencia Forestal en México, 34 (105), 37-69.

Villanueva D., J., Cerano P., J., Benavides, J. D., Stahle, D. W., Estrada A., J., Constante G., V., \& Tostado P., M. (2012). Reconstrucción de los niveles del Lago de Chapala con series dendrocronológicas de Taxodium mucronatum Ten. Revista Mexicana de Ciencias Forestales, 3(14), 55-68.

Villanueva D., J., Cerano P., J., Rosales M., S., Arrocena L., J. C., Stahle, D. W., Ruiz C., J. A., \& Martínez S., A. R. (2014). Variabilidad hidroclimática reconstruida con anillos de árboles para la cuenca alta del Río Mezquital, Durango. Revista Mexicana de Ciencias Agrícolas, Publicación Especial No. 10, 1897-2012.

Villanueva D., J., Cerano P., J., Olivares B., N.C., Valles P., M. I., Stahle, D. W., \& Cervantes, M. R. (2015). Respuesta climática del ciprés (Hesparocyparis guadalupensis) en isla Guadalupe, Baja California, México. Madera y Bosques, 21(3), 149-160. doi: 10.21829/myb.2015.213464

Wolter, K., \& Timlin, M. S. (2011). El Niño/Southern Oscillation behavior since 1871 as diagnosed in an extended multivariate ENSO index (MEI.ext). International Journal of Climatology, 31(7), 1074-1087. doi: 10.1002/joc.2336

Wright, P. B. (1979). Persistence of rainfall anomalies in the Central Pacific. Nature, 277, 371-374. doi: 10.1038/277371a0

Manuscrito recibido el: 24 de noviembre de 2016

Aceptado el: 25 de agosto de 2017

Publicado el: 8 de marzo de 2018

Este documento se debe citar como:

Villanueva-Díaz, J., Rubio-Camacho, E. A., Chávez-Durán, A. A., ZavalaAguirre, J. L., Cerano-Paredes, J., \& Martínez-Sifuentes, A. R. (2018) Respuesta climática de Pinus oocarpa Schiede Ex Schetol en el Bosque La Primavera, Jalisco. Madera y Bosques, 24(1), e2411464. doi: 10.21829/myb.2018.2411464

Madera y Bosques por Instituto de Ecología, A.C. se distribuye bajo una Licencia Creative Commons Atribución-NoComercialCompartirlgual 4.0 Internacional. 